\title{
How eruptions of a small filament feed materials to a nearby larger-scaled filament
}

\author{
H. Wei, Z. Huang, Z. Hou, Y. Qi, H. Fu, B. Li and L. Xia \\ ${ }^{1}$ Shandong Provincial Key Laboratory of Optical Astronomy and Solar-Terrestrial Environment, Institute of Space Sciences, \\ Shandong University, Weihai, 264209 Shandong, China. \\ ${ }^{2}$ School of Space Sciences and Physics, Shandong University, Weihai, 264209 Shandong, China.
}

Accepted XXX. Received YYY; in original form ZZZ

\begin{abstract}
As one of the most common features in the solar atmosphere, filaments are significant not only in the solar physics but also in the stellar and laboratory plasma physics. With the New Vacuum Solar Telescope and the Solar Dynamics Observatory, here we report on multi-wavelength observations of eruptions of a small $\left(30^{\prime \prime}\right)$ filament (SF) and its consequences while interacting with the ambient magnetic features including a large $\left(300^{\prime \prime}\right)$ filament $(\mathrm{LF})$. The eruptions of the SF drive a two-side-loop jet that is a result of magnetic reconnection between the SF threads and an over-lying magnetic channel. As a consequence of the eruption, the heating in the footpoints of the SF destabilises the barbs of the LF rooted nearby. Supersonic chromospheric plasma flows along the barbs of the LF are then observed in the $\mathrm{H} \alpha$ passband and they apparently feed materials to the LF. We suggest they are shock-driven plasma flows or chromospheric evaporations, which both can be the consequences of the heating in the chromosphere by nonthermal particles generated in the magnetic reconnection associated with the two-side-loop jet. Our observations demonstrate that the destabilisation in the vicinity of the footpoints of a barb can drive chromospheric plasma feeding to the filament.
\end{abstract}

Key words: Sun:atmosphere - Sun: filaments - methods: data analysis

\section{INTRODUCTION}

Solar filaments (named prominences while they are appearing near and extending above the solar limb) are one of the most common features in the solar atmosphere. They are dense, cool and partially-ionized plasma structures that anchor to the photosphere and extend outwards into the Sun's hot corona. They can be clearly seen in the chromospheric emissions, such as $\mathrm{H} \alpha$ passband. Their maintenance in and interaction with the fully-ionized corona can be of applications in the laboratory plasma. They might undergo instability and eruption that can develop into solar flares and/or coronal mass ejections (Wang 2006; Chen 2011; Shibata \& Magara 2011; Parenti 2014; Huang et al. 2014; Benz 2017), thus are of significance in the space weather. Although solar filaments have been intensively studied for decades, many puzzles remain unsolved because of the complexity of these phenomena (Parenti 2014; Gibson 2018). Observations with better and better resolution actually add more and more puzzles surrounding the phenomena (Gibson 2018), because of their high variability that each individual

* E-mail: z.huang@sdu.edu.cn can show different dynamics especially in fine scale at the limit of the instrumental resolution (Parenti 2014).

As a critical puzzle in the physics of solar filaments, their formation and maintenance have attracted attentions of numerous studies. It has been well established that solar filaments are formed in filament channels along polarity inversion lines (see the reviews by e.g. Martin 1998; Mackay et al. 2010; Parenti 2014; Gibson 2018). Filament channels are regions where the chromospheric fibrils are aligned with the polarity boundary. They are believed to be the prerequisite for filament formation (e.g. Gaizauskas et al. 1997; Okamoto et al. 2009; Buehler et al. 2016). They are voids before filament materials filled in and thus not visible in the remotesensing images (see Martin 1998, and references therein). The magnetic structures of filament channels are believed to be twisted or sheared arcades and thus provide dips to support filament plasmas (van Ballegooijen \& Martens 1989; DeVore \& Antiochos 2000; Gaizauskas et al. 2001; van Ballegooijen 2004; Wang \& Muglach 2007; Su et al. 2010; Jiang et al. 2014; Yan et al. 2015). Therefore, there are two critical aspects in understanding the formation of solar filaments. How is the magnetic structure of a filament 
channel formed? How are the cool plasmas transferred into there?

Many efforts have been put on understanding the formation of solar filaments (see the recent reviews by Parenti 2014; Gibson 2018). How a solar filament is formed can be different from case to case (even different from time to time in a particular case), but some basic processes usually occur, including emergence of twisted flux tubes (see e.g. Chen et al. 2018; Liu et al. 2019), shearing motion parallel to the polarity inversion line (see e.g. Chae et al. 2001; Yan et al. 2015; Li et al. 2018), magnetic reconnection among pre-existed (and newly-emerged) magnetic structures (see e.g. Yang et al. 2016; Zhou et al. 2016; Wang et al. 2017; Xue et al. 2017; Chen et al. 2018), magnetic cancellation at the polarity boundary (see e.g. Martin et al. 1985; Martin 1990; Gaizauskas et al. 1997; Chae et al. 2000; Wang 2001; Chae 2003; Wang \& Muglach 2013). These processes can link to the formation of and/or material supplement to a filament channel.

How materials are supplied to a filament channel has been a hot topic in the community, especially in the past decade when more and more advance instruments are accomplished. The sources of filament materials are different from case to case (see Parenti 2014, and references therein). Two well-known scenarios include coronal source (i.e. coronal condensation) and chromospheric source (i.e. cool plasma injection, see e.g. Karpen et al. 2006; Song et al. 2017). For any scenario, how the material supplement to a filament channel takes place remains inconclusive. Here we summarize a few studies on how cool plasmas are transferred from chromosphere to a filament channel. Chae et al. (2000) observed a transient flow field in a system of small $\mathrm{H} \alpha$ loops that some merge into a filament, and the observed evolution of the chromospheric flows agrees with a scenario that magnetic reconnection is a way of cool plasma injection in a filament as is proposed by such as Priest et al. (1996). Chae (2003) reported that a series of jets and small eruptions were taking place during the formation of the filament. Liu et al. (2005) found that chromospheric surge materials were injected from one terminal along the main axis of the filaments or the filament channels and played an important role in the formation of the filaments. Zou et al. (2016) and Zou et al. (2017) found that filaments are maintained by the continuous injection of cold chromospheric plasma along the filament threads. Wang et al. (2018) observed the formation of a filament via cool materials with a temperature of about $10^{4} \mathrm{~K}$ ejected by a series of chromospheric jets seen in $\mathrm{H} \alpha$ images. Their observations also suggest that the jets are results of magnetic reconnection between pre-existed magnetic fields and newly-emerged magnetic fields. Furthermore, Wang et al. (2019) reported in another case that the jets forming the filament consist of both cool chromospheic jets and warmer jets seen in He II $304 \AA$. A recent study with numerical experiment (Zhou et al. 2020) found that turbulences in the chromosphere can drive plasma evaporations that feed materials to a filament.

Here, we report on multi-wavelength observations of the dynamics of active-region filaments in $\mathrm{H} \alpha$ and extreme Ultraviolet (EUV) passbands. In this case eruptions of a small filament and the consequences of its interactions with the ambient magnetic structures result in magnetic reconfiguration in the region and material supplement to a nearby large filament. In what follows, we give the data description in Section 2, the results and discussion in Section 3 and the conclusions in Section 4.

\section{OBSERVATIONS}

The observations were taken on March 142013 with a target of AR NOAA 11692. The data were achieved by the ground-based 1-m New Vacuum Solar Telescope (NVST, Liu et al. 2014) of Yunnan Astronomical Observatories of China, and the space-born Atmospheric Imaging Assembly (AIA, Lemen et al. 2012) and the Helioseismic and Magnetic Imager (HMI, Schou et al. 2012) onboard the Solar Dynamics Observatory (SDO, Pesnell et al. 2012).

The NVST data include a series of filtergram images taken at the centre of $\mathrm{H} \alpha$ line with a bandpass of $0.25 \AA$. The data have been fully calibrated and reconstructed (see details in Xiang et al. 2016), and the cadence of the time series is about 12 seconds. The stabilisation of the image series was carried out by a fast sub-pixel image registration algorithm (Feng et al. 2012; Yang et al. 2015). The spatial size of a pixel of the $\mathrm{H} \alpha$ images is $0.17^{\prime \prime}$.

The images taken with the AIA passbands of $1600 \AA$, $304 \AA, 171 \AA$, $193 \AA, 211 \AA, 335 \AA, 131 \AA$ and $94 \AA$ are analysed. The AIA data have a spatial resolution of $1.2^{\prime \prime}$, and a cadence of $24 \mathrm{~s}$ for the $1600 \AA$ passband and $12 \mathrm{~s}$ for the rest. The analysed HMI line-of-sight magnetograms have a cadence of $45 \mathrm{~s}$ and a spatial resolution of $1.2^{\prime \prime}$.

The images from different instruments and passbands have been aligned by using multiple reference structures (such as sunspots, bright dots, filament threads, etc.) in passbands with closest representative temperatures.

\section{RESULTS AND DISCUSSION}

In Figure 1 and the associated animation, we show the evolution of the region of interest as seen in HMI, NVST and AIA. We can see a large filament (LF) with an apparent length of about $300^{\prime \prime}$ extending in the south-north direction, which is clearly shown in $\mathrm{H} \alpha$ and AIA $304 \AA$ and $171 \AA$ (see the cyan lines that mark a few filament threads in Figure 1a1\&b1). Above the filament, we observe overlying coronal loops in the AIA $171 \AA$ images (not shown here), which connect the anti-polarity features at both sides of the filament (see the magnetic features at the sides denoted by "P1" and "N1" in Figure 1a1). It indicates that the large filament extends along the polarity inversion line (PIL) between "P1" and "N1". Such a magnetic connectivity is also confirmed by magnetic extrapolation carried out for the same active region on the next day (Kawabata et al. 2020, see their Figures 2\&4). At the west side of the LF, a small filament (SF) with an apparent length of about $30^{\prime \prime}$ is seen (see the feature denoted by yellow arrows in Figure 1b1\&d1). The SF apparently locates along the PIL between the anti-polarity features denoted by "P2" and "N2" in Figure 1a1, and it shows a twisted structure suggesting a sheared field lines (see e.g. DeVore \& Antiochos 2000). Besides the main bodies of the two filaments, we also see barbs of the LF connecting the region in the vicinity of "P2" (see the black arrows in Figure 1d1).

While tracing the evolution of the region, we observe 

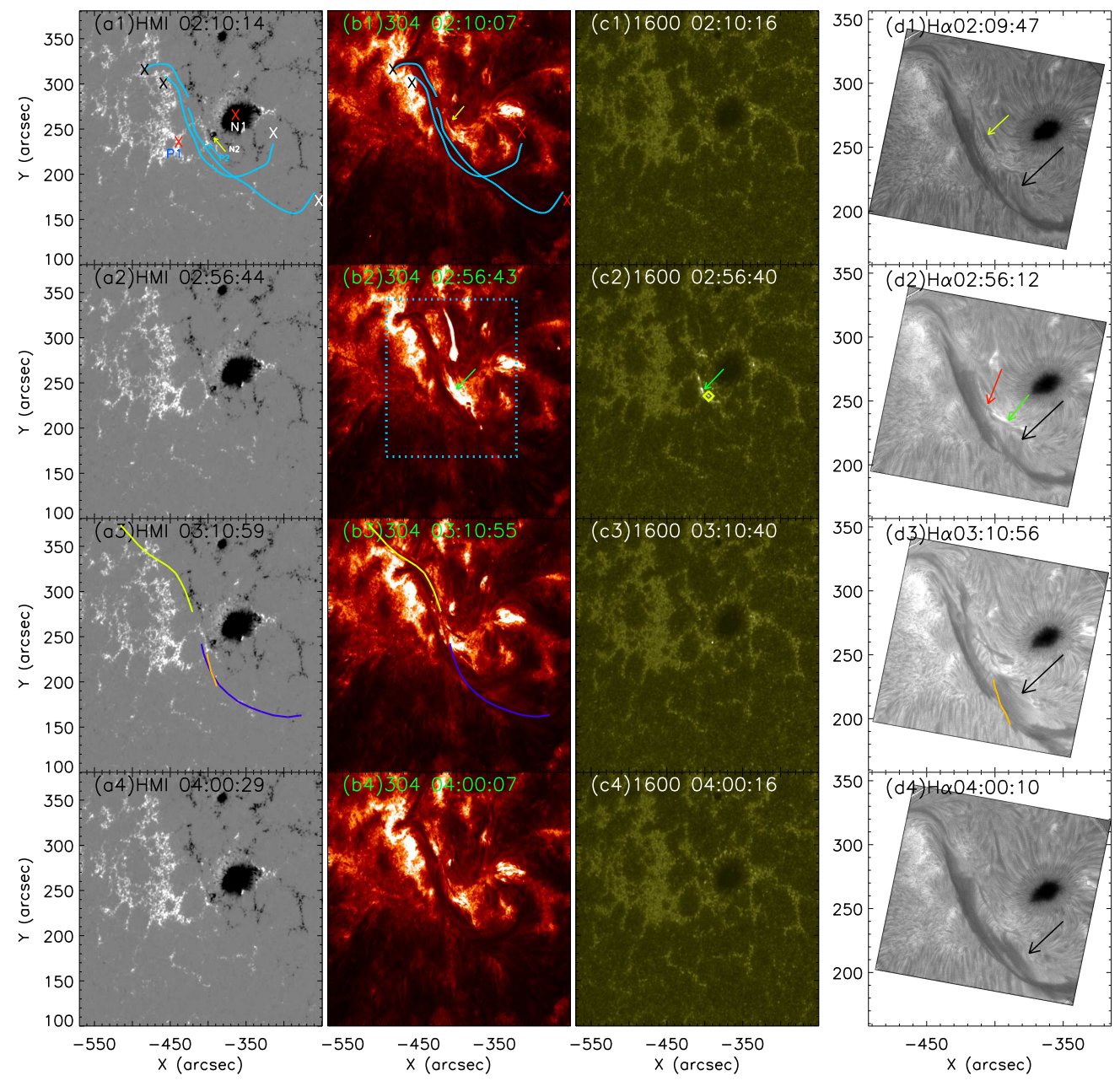

Figure 1. The region of interest viewed in the HMI line-of-sight magnetograms (a1-a4), AIA $304 \AA$ (b1-b4) AIA 1600 $\mathrm{A}$ (c1-c4) and NVST H $\alpha(\mathrm{d} 1-\mathrm{d} 4)$. The field-of-view shown in column (d) is marked in panel (b2) as the dotted-line square. The solid lines in cyan in panels (a1) and (b1) outline a few threads of the LF, and the associated polarities are marked as "P1" and "N1". The SF is denoted by yellow arrows in panels (b1) and (d1), and its associated polarities are marked as "P2' and "N2" in panel (a1). The crosses in white and black marked in panels (a1) are the visible footpoints of the LF, while the crosses in red are the footpoints of the overlying coronal loops as seen in AIA $171 \AA$ images. The red arrow in panel (d2) marks the plasma flow that might be the trigger of the SF eruption. The green arrows in panels (b2), (c2) and (d2) denote the eruption site. The black arrows in the 4th column denote the barbs of the LF. The yellow and blue lines in panels (a3) and (b3) mark the trajectories of the two-side-loop jet. The orange lines in panels (a3) and (d3) mark the trajectory of the plasma flow along the LF barbs. The square in yellow in panel (c2) marks the compact brightening that is used to produce the lightcurve shown in Figure 3b. (An online animation is provided.)

eruptions of the SF and the consequences of the interactions with the surrounding magnetic structures. The eruption starts at around 02:56 UT (see the second row of Figure 1 and the associated animation). About one minute before the eruption of the SF, we observed a flow with an apparent speed of about $50 \mathrm{~km} \mathrm{~s}^{-1}$ (supersonic) injects into the region (appearing as a dark feature in $\mathrm{H} \alpha$, see the structure denoted by the red arrow in Figure 1d2 and can be clearly followed in the associated animation). This injection might plays a role in the destabilization of the SF, but that should require further theoretical study to confirm.

The eruption of the SF shows a complex morphology in the site (see the location denoted by green arrows in Figure 1b2,c2\&d2) including multiple compact brightenings seen in AIA $1600 \AA$ observations (see Figure 1c2). Due to their compact nature, these brightenings are very likely the sites where the energy was deposited in the chromosphere via nonthermal particles generated in the eruption of the SF. Many of these brightenings are located in the vicinity of the footpoints of the barbs of the LF. To investigate the thermal structures of the erupted region, we apply a differential emission measure (DEM) analysis (Cheung et al. 2015; Su et al. 2018) on the images taken with AIA EUV passbands and obtain the emission measure (EM) of the region. In Figure 2 and the associated animation, we show zoomed-in views of the erupted region in AIA $94 \AA$ and $171 \AA$ channels and EM images at a various temperature ranges. Besides the multithermal nature of the eruption, we can see that the erupted region consists of multiple fine structures in the EM maps, which are consistent with the twisted appearance of the SF.

While the SF is lifted and moving toward the region of the LF, we observed a bi-directional jet (see the trajectories 

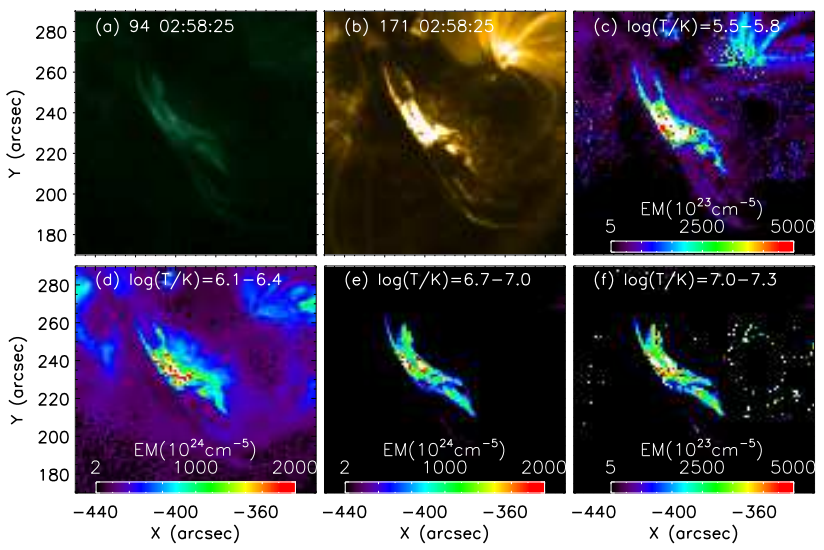

Figure 2. The eruption of the SF seen in the AIA $94 \AA$ and $171 \AA$ passbands and the derived EM maps at various temperature ranges as noted. (An online animation is provided.)
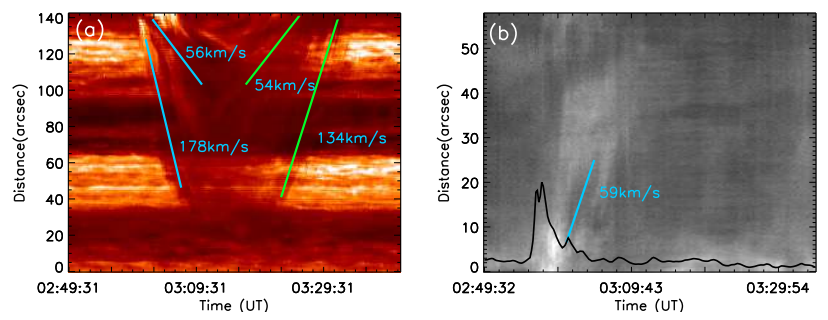

Figure 3. (a): Time-distance plots taken from the northward trajectory of the two-side-loop jet (see the yellow lines in Figure 1a3\&b3) with the AIA 304 A observations. (b): Time-distance plots of the plasma flow along the LF barbs (see the orange lines in Figure $1 \mathrm{a} 3 \& \mathrm{~d} 3$ ) with the NVST $\mathrm{H} \alpha$ observations. The apparent speeds derived from these plots are marked. The over-plotted black solid line is the AIA $1600 \AA$ lightcurve of a compact brightening located in the vicinity the footpoints of the barbs and at the end of the trajectory that used to produced this time-distance map (see the yellow square in Figure 1c2).
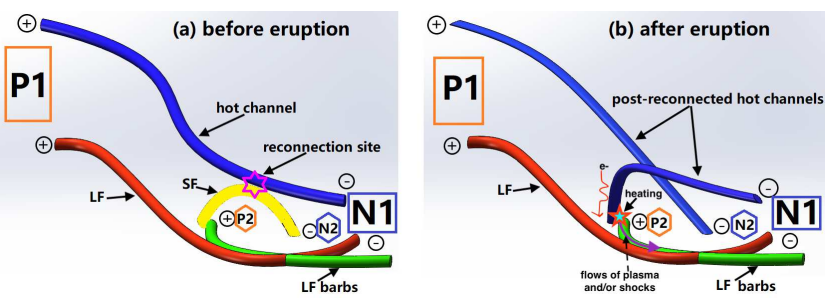

Figure 4. A scenario interprets the interaction between the erupted SF and the hot channel and how the materials are fed to the LF. (See the main text for details.)

marked by yellow and blue lines in Figure 1a3\&b3) that has been analysed in detail and interpreted as a two-side-loop jet by Yang et al. (2019). Small-scaled magnetic cancellations associated with this event have also been reported by Yang et al. (2019), suggesting disturbances in the lower atmosphere. Because we are not focus on the jet, here we show the time-distance plots along the northward trajectory of the jet (see the yellow line in Figure 1a3\&b3) as seen in AIA $304 \AA$ only (Figure 3a). In additional to Yang et al. (2019), we would like to point out that the trajectory of the jet does not follow any pre-exited $\mathrm{H} \alpha$ thread of the LF (clearly in the north part, see the yellow line in Figure 1a3\&b3 and the associated animation of Figure 1). This indicates that there is a magnetic channel laid above the LF and it is not visible in the images of $\mathrm{H} \alpha$ and AIA channels. This "invisible" channel might be an empty flux tube or a hot channel that is evidenced by the X-ray images as shown in Kawabata et al. (2020). The jet is most clearly seen with all AIA EUV passbands, and the EM analyses suggest its multi-thermal nature (see Figures 1\&2). However, it does not show response in $\mathrm{H} \alpha$ images, again suggesting its origin above the chromosphere. Moreover, we would also like to mention that the northward jet flow (yellow line in Figure 1a3\&b3) is reversed about 15 minutes after the initiation of the jet flows and has traveled for about 140" (see Figure 3a). After about 03:30 UT, the hot channel has drained and again disappears from the observations of the AIA EUV passbands. Thus, the jet does not directly feed materials into the LF.

We also clearly observe plasma flows along the barbs of the LF following to the eruption of the SF (see the black arrows in Figure 1d1-d4). The plasma flows can be clearly seen as dark features in the $\mathrm{H} \alpha$ images, and they are mostly cool plasma as seen in the EM images (see Figure 2 and the associated animation). The flows apparently follow the compact brightening in the footpoints and start as propagations of bright features (see Figure $3 \mathrm{~b}$ ). By tracing the evolution of the $\mathrm{LF}$ in $\mathrm{H} \alpha$ images, we found that these plasma flows apparently change the geometry of the LF (see the feature denoted by black arrow in Figure 1d4). Thus, we conclude that these plasma flows have fed materials to the LF. The flows have an apparent speed of about $59 \mathrm{~km} \mathrm{~s}^{-1}$ measured with the $\mathrm{H} \alpha$ time-distance plot (Figure $3 \mathrm{~b}$ ). Although we cannot rule out the possibility that such apparent flows are results of dark features drifting into the field-of-view, it is very likely that they are real plasma flows because of the following darkening of the barbs seen in the $\mathrm{H} \alpha$ images. Since the speed is much larger than the sound speed in the chromosphere, if they are real plasma flows they should present shock behaviours and a hint is that the brightenings propagate ahead of the dark features in $\mathrm{H} \alpha$ observations (see the bright structure in Figure 3b that start one to two minutes before the dark one as marked by the blue line).

Based on the observations, we give our interpretation for these processes in Figure 4. Before the eruption (Figure 4a), the LF includes its main threads (represented by the red line) connecting "P1" and "N1" and barbs connecting "P2" and "N1"; the SF connecting "P2" and "N2" is represented by the yellow line; the hot channel laid above the LF is shown as the blue line. When the SF is destabilised, its threads are lifted up and encounter the hot channel above. That triggers magnetic reconnection between the hot channel and the SF threads, which generates the twoside-loop jet flows along the reconfigured hot channels (see two blue lines in Figure 4b). We are aware of that this reconnection does not change the general configuration of the main filament, which is different from that proposed in van Ballegooijen \& Martens (1989). We believe that the magnetic reconnection produces nonthermal particles, and some of those travel along the field lines toward the chromosphere at "P2" that cause heating in the region. The heating in the chromosphere of "P2" destabilises the footpoints of the barbs of the LF and results in shock flows that drive 
chromosperic plasma feeding to the LF. Alternatively, the destabilisation in the footpoints of the barbs can cause turbulences, which can generate chromospheric evaporation and that feeds chromospheric plasma to the LF as shown in the simulation of Zhou et al. (2020).

\section{CONCLUSIONS}

In the present, we report on NVST and SDO observations of eruptions of a small filament (SF) with a size of about $30^{\prime \prime}$ and its interactions with the other magnetic structures including a large filament with a size of about $300^{\prime \prime}$ in the region. The SF and LF are above different polarity inversion lines that belong to different magnetic patches, but there are barbs of the LF connecting the patch of positive polarity of the SF. The erupted SF threads encounter a magnetic channel above the LF that is previously invisible in $\mathrm{H} \alpha$ and AIA EUV images. That produces a two-side-loop jet, which is suggested to be a result of magnetic reconnection between the SF threads and the magnetic channel. As a consequence of the eruption, plasma flows are seen in the barbs of the LF that connect the positive polarity of the SF, and these flows appear to feed materials to the LF. We interpret that the materials are fed to the LF via shock-driven plasma flows or chromospheric evaporations, which both can be the consequences of destabilisation in the chromospheric footpoint region from the heating by nonthermal particles generated in the magnetic reconnection associated with the two-side-loop jet. Our observations demonstrate that the destabilisation in the footpoints of a barb can indeed drive chromospheric plasma feeding to the filament.

\section{ACKNOWLEDGEMENTS}

We are grateful to the anonymous referee for the constructive and helpful comments. This research is supported by the Strategic Priority Program of CAS (XDA15017300), National Natural Science Foundation of China (U1831112, 41974201, 41627806, 11761141002, 41674172), and the Young Scholar Program of Shandong University, Weihai (2017WHWLJH07). We would like to thank the NVST operation team for preparation of the data. Courtesy of NASA/SDO, the AIA and HMI teams and JSOC.

\section{DATA AVAILABILITY}

The data analysed in this study can be freely requested from the official websites at http://fso.ynao.ac.cn (NVST) and http://jsoc.stanford.edu (SDO) using the information given in the main text.

\section{REFERENCES}

Benz A. O., 2017, Living Reviews in Solar Physics, 14, 2

Buehler D., Lagg A., van Noort M., Solanki S. K., 2016, A\&A, 589, A31

Chae J., 2003, ApJ, 584, 1084

Chae J., Denker C., Spirock T. J., Wang H., Goode P. R., 2000, Sol. Phys., 195, 333
Chae J., Wang H., Qiu J., Goode P. R., Strous L., Yun H. S., 2001, ApJ, 560, 476

Chen P. F., 2011, Living Reviews in Solar Physics, 8, 1

Chen H., Yang J., Yang B., Ji K., Bi Y., 2018, Sol. Phys., 293, 93

Cheung M. C. M., Boerner P., Schrijver C. J., Testa P., Chen F., Peter H., Malanushenko A., 2015, ApJ, 807, 143

DeVore C. R., Antiochos S. K., 2000, ApJ, 539, 954

Feng S., Deng L., Shu G., Wang F., Deng H., Ji K., 2012, in 2012 IEEE Fifth International Conference on Advanced Computational Intelligence (ICACI). pp 626-630, doi:10.1109/ICACI.2012.6463241

Gaizauskas V., Zirker J. B., Sweetland C., Kovacs A., 1997, ApJ, 479,448

Gaizauskas V., Mackay D. H., Harvey K. L., 2001, ApJ, 558, 888 Gibson S. E., 2018, Living Reviews in Solar Physics, 15, 7

Huang Z., Madjarska M. S., Koleva K., Doyle J. G., Duchlev P., Dechev M., Reardon K., 2014, A\&A, 566, A148

Jiang C., Wu S. T., Feng X., Hu Q., 2014, ApJ, 786, L16

Karpen J. T., Antiochos S. K., Klimchuk J. A., 2006, ApJ, 637,531

Kawabata Y., Inoue S., Shimizu T., 2020, The Astrophysical Journal, 895, 105

Lemen J. R., et al., 2012, Sol. Phys., 275, 17

Li D., Shen Y., Ning Z., Zhang Q., Zhou T., 2018, ApJ, 863, 192

Liu Y., Kurokawa H., Shibata K., 2005, ApJ, 631, L93

Liu Z., et al., 2014, Research in Astronomy and Astrophysics, 14,705

Liu L., Cheng X., Wang Y., Zhou Z., 2019, ApJ, 884, 45

Mackay D. H., Karpen J. T., Ballester J. L., Schmieder B., Aulanier G., 2010, Space Sci. Rev., 151, 333

Martin S. F., 1990, Conditions for the Formation of Prominences as Inferred from Optical Observations. p. 1, doi:10.1007/BFb0025641

Martin S. F., 1998, Sol. Phys., 182, 107

Martin S. F., Livi S. H. B., Wang J., 1985, Australian Journal of Physics, 38, 929

Okamoto T. J., et al., 2009, ApJ, 697, 913

Parenti S., 2014, Living Reviews in Solar Physics, 11, 1

Pesnell W. D., Thompson B. J., Chamberlin P. C., 2012, Sol. Phys., 275, 3

Priest E. R., van Ballegooijen A. A., Mackay D. H., 1996, ApJ, 460,530

Schou J., et al., 2012, Sol. Phys., 275, 229

Shibata K., Magara T., 2011, Living Reviews in Solar Physics, 8,6

Song H. Q., et al., 2017, ApJ, 836, L11

Su Y., van Ballegooijen A., Golub L., 2010, ApJ, 721, 901

Su Y., Veronig A. M., Hannah I. G., Cheung M. C. M., Dennis B. R., Holman G. D., Gan W., Li Y., 2018, ApJ, 856, L17

Wang Y. M., 2001, ApJ, 560, 456

Wang J., 2006, Advances in Space Research, 38, 1887

Wang Y. M., Muglach K., 2007, ApJ, 666, 1284

Wang Y. M., Muglach K., 2013, ApJ, 763, 97

Wang J., Yan X., Qu Z., Xue Z., Yang L., 2017, ApJ, 839, 128

Wang J., Yan X., Qu Z., UeNo S., Ichimoto K., Deng L., Cao W., Liu Z., 2018, ApJ, 863, 180

Wang J., Yan X., Guo Q., Kong D., Xue Z., Yang L., Li Q., 2019, MNRAS, 488, 3794

Xiang Y.-y., Liu Z., Jin Z.-y., 2016, New Astron., 49, 8

Xue Z., Yan X., Yang L., Wang J., Zhao L., 2017, ApJ, 840, L23

Yan X. L., Xue Z. K., Pan G. M., Wang J. C., Xiang Y. Y., Kong D. F., Yang L. H., 2015, ApJS, 219, 17

Yang Y.-F., Qu H.-X., Ji K.-F., Feng S., Deng H., Lin J.-B., Wang F., 2015, Research in Astronomy and Astrophysics, 15, 569

Yang B., Jiang Y., Yang J., Yu S., Xu Z., 2016, ApJ, 816, 41

Yang B., Yang J., Bi Y., Xu Z., Hong J., Li H., Chen H., 2019, The Astrophysical Journal, 887, 220

Zhou G., Wang J., Zhang J., 2016, Sol. Phys., 291, 2373 
Zhou Y. H., Chen P. F., Hong J., Fang C., 2020, Nature Astronomy,

Zou P., Fang C., Chen P. F., Yang K., Hao Q., Cao W., 2016, ApJ, 831, 123

Zou P., Fang C., Chen P. F., Yang K., Cao W., 2017, ApJ, 836,122

van Ballegooijen A. A., 2004, ApJ, 612, 519

van Ballegooijen A. A., Martens P. C. H., 1989, ApJ, 343, 971

This paper has been typeset from a $\mathrm{T}_{\mathrm{E}} \mathrm{X} / \mathrm{LAT}_{\mathrm{E}} \mathrm{X}$ file prepared by the author. 